\title{
Calidad educativa en la Universidad Metropolitana del Ecuador a lo largo de un lustro
}

\section{Educational quality in the Metropolitan University of Ecuador along one half a decade}

\author{
Somaris Fonseca Montoya ${ }^{1}$ \\ sfonseca@umet.edu.ec \\ http://orcid.org/0000-0003-0070-9741 \\ Reinaldo Requeiro Almeida ${ }^{2}$ \\ rrequeiro@ucf.edu.cu \\ http://orcid.org/0000-0001-8609-5554
}

Recibido: 1/4/2020; Aceptado: 1/6/2020

\section{RESUMEN}

El estudio consiste en una revisión documental, de publicaciones resultantes de los proyectos de investigación, Mejoramiento de la Calidad Educativa de la Educación Básica Ecuatoriana y el proyecto Gestión de la Calidad de la Educación, uno y otro desarrollados de manera continua e ininterrumpida entre 2014-2016 y 2017-2019, respectivamente. Las aportaciones de ambos proyectos, se han hecho visibles en revistas científicas, no obstante, ellas admiten un análisis sistematizado, que, de paso ayude a mejorar la concepción de los procesos de investigación en la Universidad Metropolitana del Ecuador en su sentido más amplio. Puede señalarse que las fortalezas encontradas en la trayectoria de los proyectos antes referidos radican, en lo fundamental, en haber ayudado a la apertura de un pensamiento de cooperación interprofesional, la capacidad de convocatoria institucional, las contribuciones metodológicas, así como la cooperación interprofesional en un ámbito de valoraciones múltiples, todo lo cual, se ha manifestado con sostenibilidad en el tiempo.

Palabras clave: Proyecto de investigación, producción científica, contribuciones metodológicas, cooperación interprofesional, valoraciones múltiples

\section{ABSTRACT}

The study consists of a documentary review of publications resulting from the research projects: Improvement of the Educational Quality of Basic Ecuadorian Education and the Management of the Quality of Education project, both developed in a continuous and uninterrupted, between 2014- 2016 and 2017-2019 respectively. The contributions of both projects have been made visible in scientific journals, however, they admit a systematized analysis, which, incidentally, helps to improve the conception of research processes at the Metropolitan University of Ecuador in its broadest sense. It can be pointed out that the strengths found in the trajectory of the aforementioned projects, lie essentially in having helped to open up inter-

\footnotetext{
${ }^{1}$ Universidad Metropolitana del Ecuador. Matriz Guayaquil, Ecuador. Universidad de Cienfuegos., Cuba

${ }^{2}$ Universidad Metropolitana del Ecuador. Matriz Guayaquil, Ecuador. Universidad de Cienfuegos, Cuba.
} 


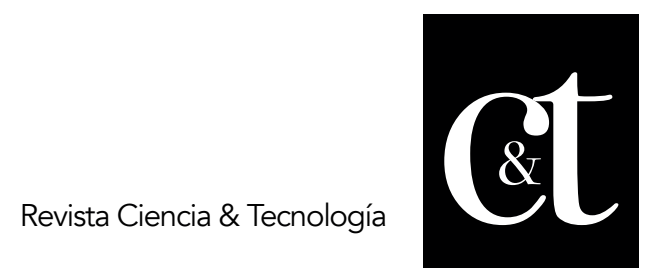

No. 27, 31 de julio de 2020

ISSN impreso: 1390 - 6321

ISSN online: 2661 - 6734

professional cooperation thinking, institutional convening capacity, methodological contributions, as well as inter-professional cooperation in a field of evaluations multiple, all of which have manifested steadily over time.

Keywords: Research project, scientific production, methodological contributions, interprofessional cooperation, multiple valuations

\section{Introducción}

Si bien en el contexto de la Educación Superior ecuatoriana, la Universidad Metropolitana del Ecuador se ha venido consolidando en los últimos tiempos como una institución de creciente desarrollo, en la mejora de sus índices generales de acreditación ha influido, de manera particular, la formación continua de sus docentes. La concurrencia a sus predios de un claustro portador de un saber multinacional, ha contribuido también a reforzar, la proyección fundacional de dicho centro, signado por un carácter marcadamente inclusivo y que se basa en un proyecto educativo desarrollador que maneja la incorporación y retención a estudiantes de los más diversos sectores sociales, en plenitud de atención al ejercicio de sus oportunidades. Un alumnado diverso, es atendido por un claustro docente, con enriquecidas herramientas profesionales, que garantizan disímiles posibilidades para asumir los retos que comporta el tratamiento a semejante pluralidad, esto hoy se hace visible en diferentes publicaciones científicas desarrolladas por los propios docentes de la UMET.

Basado en lo anterior, el presente artículo de revisión analiza una muestra de las salidas de resultados, publicados todos en los últimos cinco años en las siguientes Revistas científicas:

- Universidad y Sociedad,

- Revista Conrado y

- Revista Metropolitana de Ciencias Aplicadas (REMCA).

Como parte del análisis debe indicarse que, la mayor contribución del proyecto Gestión de la Calidad de la Educación, reside no solo en la copiosa producción científica de los investigadores que se encuentran participando directamente en sus tareas, sino en haber abierto un horizonte de discernimiento que ha movilizado, mediante la socialización, a otros muchos colegas para asumir una práctica investigativa orientada a la búsqueda de la calidad.

Puede hablarse al efecto de una capacidad de convocatoria institucional tangible desde el mismo proyecto, contribuciones metodológicas, cooperación interprofesional, valoraciones múltiples de los resultados.

\section{Desarrollo}

Particular mención requiere en este análisis, el afrontamiento a los problemas de salud tanto del estudiantado como de los profesores, cual condición básica para los aprendizajes, en tal caso, las relaciones morbodidactogénicas que acontecen al interior del ejercicio profesional, pueden resumirse en afectaciones que reciben los docentes en su salud, como consecuencia de una práctica profesional angustiosa. 


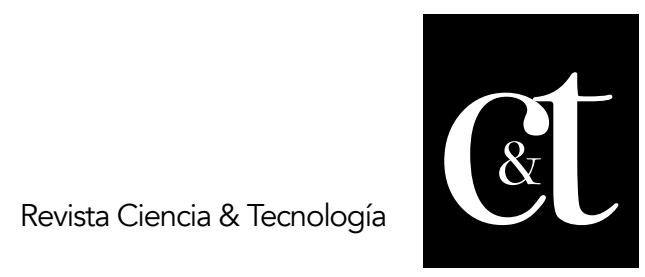

No. 27, 31 de julio de 2020

ISSN impreso: 1390 - 6321

ISSN online: 2661 - 6734

Tales angustias son originadas, de modo predominante, por la insuficiente preparación para ejercer un magisterio menos agotador, el empleo de métodos de enseñanza que no concitan el interés y la cooperación estudiantil, se sabe de antemano que, semejantes atributos no permiten radicalizar un aprendizaje cooperativo y liberador del esfuerzo laboral docente. Entre otros factores se añaden las condiciones físicas del puesto de trabajo, las relaciones con los compañeros y los administrativos, en tanto, todo esto repercute en el sentido más amplio de las oportunidades para emprender un desempeño exitoso.

Lo anterior está en correspondencia con los propios fines que se plantea la Universidad, es decir: la formación de profesionales bien calificados y la generación del nuevo saber proveniente de la actividad científica, en su articulación pregrado postgrado. Con ello la gestión de la calidad de la Educación se amplía, ya que se deciden los precedentes de un contexto de oportunidades, para ordenar la misión universitaria con una orientación desarrolladora hacia las exigencias ocupacionales, pero estructuradas de manera saludable.

Dicho proceder se ratifica en la presentación de una oferta académica asentada en procesos diagnósticos, llevados adelante en el entorno social, en tanto la investigación propicia la ayuda notoria a la calidad de los diferentes procesos sustantivos del trabajo universitario. Los resultados se evidencian en ejemplos diversos, como los proyectos de investigación y de vinculación con la sociedad, la superación docente, y su perfeccionamiento, de manera particular, el cuidado de la salud tanto de estudiantes como de trabajadores.

\section{El enfoque en la salud para una mejor calidad educativa}

El enfoque en la salud tiene mucho que ver con la calidad educativa. Mucho contribuye la atención a la educación para la salud, con una proyección preventiva. Por ejemplo, deben considerarse importantes tópicos como los relacionados a las adicciones. Tópicos como este no son asumidos en los lugares de trabajo, por lo que las universidades tienen una gran responsabilidad en llevar a cabo estrategias adecuadas. Con esto se revela una senda educativa de absoluta pertinencia investigativa. Se contribuye así a una educación de mejor calidad, al ser un proceso integral.

Como parte de estos análisis enfocados al tema de la salud en el ámbito universitario, se tiene el trabajo de (Alvarez, I., Macías, A. y Bernal, R., 2019) cuyo trabajo se enmarca en la orientación educativa a través de Talleres dirigidos a la familia, dicha propuesta, contribuye a modificar estilos de vida familiar apoyados en la previa identificación de conductas y hábitos. Aportar estas alternativas para familias de niños que estudian en un centro de educación prescolar, contribuye a que puedan acogerse a formas de intervención mediante los talleres, dado su carácter dinámico y la manera de generar experiencias de valor. No puede dejarse de lado el modo en que la educación familiar, resulta también incidente en la salud y la calidad de vida del docente, el reconocimiento de la preponderancia familiar, sin la cual no puede haber una educación de calidad y es ahí cuando se reactivan las relaciones de satisfacción de los profesionales para, poder obtener un trabajo de mayor calidad de los aprendizajes.

La contribución del trabajo en función de la orientación educativa, se revalida como fortaleza para transformar estilos de vidas no saludables, con lo que se aportan 


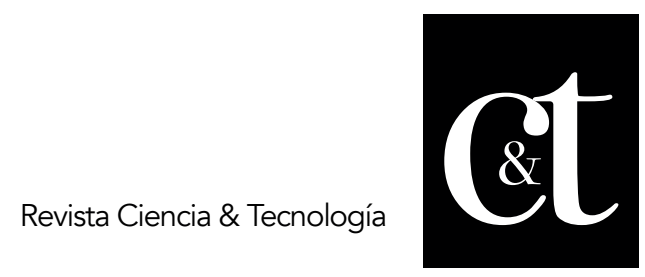

No. 27, 31 de julio de 2020

ISSN impreso: 1390 - 6321

ISSN online: 2661 - 6734

pautas para revertir situaciones educativas inconvenientes, por todo, es notoria en la obra la concesión de valoraciones múltiples que, se relacionan con un ámbito analítico, mucho más amplio de las problemáticas educativas.

Con relación a este proceso los resultados de (Montero, C. ,Porraspita, D. y Hernández, D., 2016), devela que los análisis son enmarcados en la formación investigativa estudiantil, elemento que resulta determinante para el desarrollo del currículo de tercer nivel, orientado desde el marco legal y metodológico que sustenta la política de formación.

En el trabajo subyace la contribución que ello tiene al buen estado de la salud mental, al tener que afrontar un ejercicio de formación tan complicado como la investigación y que tiene importancia para el desempeño en la práctica profesional de los educandos. El proceso de investigación de los estudiantes cual componente de su formación, dada su naturaleza les impone relativos niveles de estrés. Influye la endeblez de las herramientas metodológicas para desarrollarlo creativamente, y el desafío redunda en contra de la calidad de vida. Puede afirmarse que este artículo, fundamenta aspectos cruciales del desarrollo de conocimientos, habilidades y valores para la actividad profesional crítica; por todo se aviene con las tendencias de producción científica de la Universidad Metropolitana de Ecuador (UMET) y evidencia un notorio marco de cooperación interprofesional, en su concreción.

\section{Desarrollo de las habilidades investigativas}

El tema de las habilidades investigativas en los estudiantes, también ha sido objeto de estudio de otros investigadores de la UMET, cítese a ( López Calvajar, Toledo Rodríguez , \& Meza Salvatierra, 2017), autores que analizan el proceso de formación de habilidades para la investigación estudiantil, como requisito indispensable para el egreso en la Universidad Metropolitana del Ecuador. Los estudiantes de las carreras de Gestión Empresarial y Ciencias Administrativas y Contables fueron beneficiarios de un estudio de caso integral, desde la asignatura Metodología de la Investigación Científica. Se presenta el correspondiente diseño que ofrece contribuciones referenciales para otros similares que puedan ser realizados y en adición, una estrategia para la aplicación y su corroboración mediante resultados empíricos. En este caso se trata la modelación de alternativas pedagógicas al tomar como base el tratamiento de problemas profesionales de la práctica laboral y, en consecuencia, se ofrece un modelo orientado a dichos fines.

\section{Las prácticas pre profesionales}

Habría que subrayar la fundamentación que se hace en el trabajo respecto a que la práctica pre profesional, tal como lo destacan algunos autores (Sánchez, V., López, M. M. y Baute, M., 2014) quienes enfatizan que, "...la Práctica Pre profesional planificada permite cumplir con su encargo desarrollador, cual condición imprescindible para concretar el proceso de titulación". (Sánchez, López, \& Baute, 2014).

La gestión de la calidad de la educación, en este sentido, permite al estudiante sistematizar un conjunto de experiencias profesionales que, ayuda a implementar diferentes saberes en función de su desarrollo profesional con alta implicación en la salud, la adaptación laboral y su calidad de vida. 


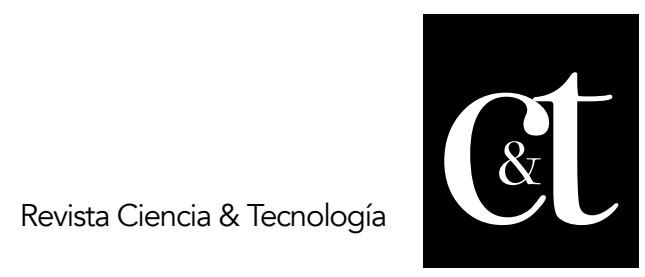

No. 27, 31 de julio de 2020

ISSN impreso: 1390 - 6321

ISSN online: 2661 - 6734

Factibles soluciones a las tareas de aprendizaje y del desempeño, pueden ser halladas, por esta vía, en correspondencia con las exigencias del modelo educativo que rige la formación del egresado, la cual, cada día más, opera en estrecha relación con una dinámica demanda social.

Gradualmente estas interacciones que reserva la práctica pre profesional, se revelan en la contrastación con la teoría, cuestión que permite el acceso a saberes afines con el desarrollo de competencias pedagógicas para el ejercicio de una Educación Inicial de actualidad.

\section{Resultados}

Los resultados obtenidos permiten nominalizar, con indiscutible claridad, seis impactos fundamentales que abarcan áreas muy diversas y que van desde el ámbito sociocultural al ambiental, los impactos tecnológicos, las valoraciones de orden económico, puede afirmarse, una vez analizado este trabajo en su integridad que se trata de una obra en la cual se sostienen valoraciones múltiples del objeto de estudio. Utilizar los soportes virtuales se fundamenta, en el trabajo, como una condición de calidad de los aprendizajes, un factor determinante para el proceso de enseñanza, en consecuencia, la inevitabilidad de su empleo dado que, cada vez más, los estudiantes se hayan influidos por la tecnología y hacen requerir en el nivel universitario de investigaciones producidas en los contextos de los proyectos que funcionan con este fin.

Tanto el trabajo precedente como otros (Chou Rodríguez, Valdés Guada, \& Sánchez Gálvez, 2017) aportan una lógica funcional a los problemas vinculados a la gestión de la calidad de la educación. Este último trabajo hace destacar el modo en que las investigaciones están destinadas a favorecer la realidad. Se requiere, señalan los autores, "mayor sistematicidad en el estudio continuo de cada módulo, en lo que respecta a la estructura y contenidos que van cambiando los entornos donde tiene lugar el proceso educativo, enfocado a mejorar la formación de competencias digitales en docentes universitarios" (Chou Rodríguez, Valdés Guada, \& Sánchez Gálvez, 2017).

Particulares contribuciones de investigaciones concernientes a la promoción de salud, por ejemplo, deparan el proceso de determinación de categorías, principios y acciones a desarrollar, para responder al problema de la implementación de tareas en la carrera de Ciencias de la Educación, evidenciando su efectividad en relación con una cultura ambiental, atemperada a las exigencias actuales de la educación superior ecuatoriana. (Pérez Payrol, Baute Roales, \& Luque Espinoza de los Monteros, 2017). Justificada capacidad de convocatoria institucional depara el proyecto de investigación Gestión de la Calidad de la Educación en la UMET, en tanto produce también contribuciones metodológicas y por tanto de cooperación interprofesional, incluso, como es el caso del trabajo recién citado, en el cual cooperan docentes de centros de educación superior de otros países, en un proceso de franca internacionalización.

Justificado resulta, en este contexto, el sistema de trabajo propuesto por, (Tamayo, M., Gil, J.L. y Carrillo, J. J, 2019), en tanto estimula la implicación institucional de modo particular en lo que conciernen a saberes relacionados con gestión empresarial, con énfasis en la atención a las demandas del desempeño individual y a partir de la mirada analítica de los estudiantes. 


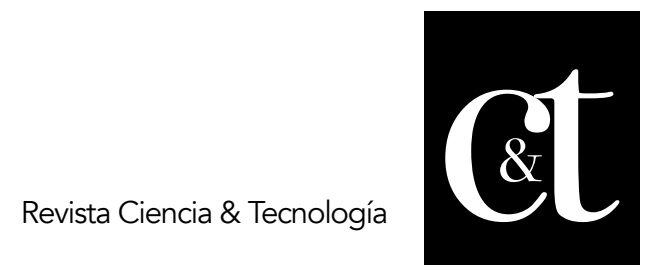

No. 27, 31 de julio de 2020

ISSN impreso: 1390 - 6321

ISSN online: 2661 - 6734

El trabajo enfatiza en el positivo efecto del acompañamiento personalizado y directo al alumnado, el cual puede contribuir mediante la atención temprana a la mejora de los aprendizajes y de los comportamientos profesionales, con énfasis en el componente actitudinal, como elemento cardinal del proceso pedagógico. Una visión muy particular de la tutoría desde la carrera y en su aserción grupal deja claro, los distintos momentos que comprende el desarrollo profesional, las vías que se asumen para diversificar su enfoque y también el modo en que el docente debe dotarse de las herramientas para conducir tan importante proceso. La concepción tutorial de la carrera Administración de Empresas, se apoya en el permanente intercambio entre los diferentes funcionarios que intervienen en la conformación final de la matrícula, ( Toledo Rodríguez, Varela, \& Meza Salvatierra, 2017).

Sobre dicho asunto se devela una amplia gama de complejidades tutoriales, por lo que se proyectan los análisis con una visión integradora que toma en cuenta, desde el personal de admisiones, que se encarga de tener el primer contacto con los estudiantes hasta el personal docente de más alta calificación.

Consecuentemente, se deriva un sistema de trabajo que permite atender tanto necesidades de orden individual como grupal de los educandos, a lo largo de las diferentes etapas de desarrollo profesional. Igualmente valioso resulta el trabajo de (Suárez, G., León, J. L., Morales, M. y Curbeira, D, 2019) en el que se presenta una modelación prevista con gradualidad, en secuencia de etapas y fases integradoras y sostenidas en un funcionamiento sistémico, en el ámbito de la formación de valores.

Resaltan en la propuesta las concepciones humanistas, por extensiones inclusivas, congruentes con los más avanzados presupuestos de la política universitaria, concebidos con dinamismo y flexibilidad, tales atributos permiten dotar al docente de una preparación ajustada a la ejecutoria del complejo proceso de la formación de valores en el ámbito de la Educación Superior. En la obra presentada por ( López Calvajar, Nelson , \& Baute, La tarea docente integradora. Caso optimización del plan de producción, 2017) referida a la integración de conocimientos interdisciplinarios en la ejecutoria del plan de estudio, puede distinguirse un basamento integrador, respecto a la importancia de la tarea docente en el proceso de formación de profesionales de las ciencias económicas y otras especialidades afines.

Su objetivo radica en el desarrollo de competencias para la elaboración del presupuesto empresarial, toda vez que la toma de decisiones relacionadas se revela como uno de los primordiales problemas que afectan hoy al completamiento del proceso de formación. No puede dejarse de mencionar como parte de esta producción lo aportado por (Millo , V., Fuentes, D. y González, V. E., 2017). Con la investigación se completa un Manual de procedimientos que contribuye a la realización de los procesos de auditoría interna que tienen lugar de manera sistemática en la Universidad Metropolitana del Ecuador. Estos resultados permiten sistematizar la metodología para la realización de las auditorías internas con lo que se contribuye, a propiciar su carácter imparcial conforme al empleo de procedimientos estandarizados. Se utiliza el manual diseñado una vez aprobado por el Consejo Académico Superior.

Puede destacarse que el aporte, atestigua la cooperación interprofesional y de conformidad con la realización de valoraciones múltiples, que contribuyen a 


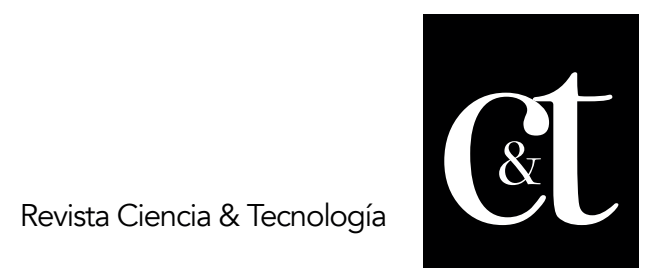

No. 27, 31 de julio de 2020

ISSN impreso: 1390 - 6321

ISSN online: 2661 - 6734

retroalimentar la calidad del servicio general de la UMET, desde el desempeño investigativo y mediante la concepción de un proyecto, adecuadamente estructurado. Mediante similares procederes se concreta el trabajo de (Jiménez, J. C. y Moreira, Y, 2019) quienes analizan el modo en que la práctica educativa inclusiva de la Universidad Metropolitana del Ecuador, deviene una alternativa en función de la satisfacción de los sujetos, en tanto la carrera y el grupo se constituyen en objetos de la evaluación del impacto del Bienestar Estudiantil.

Los significados de este proceso en la vida social e incluso en las relaciones interpersonales de nuestra época, visto a la luz de la realidad de hoy no dejan de tener impactos significativos en la educación.

\section{Conclusiones}

Luego de la revisión realizada puede concluirse que, existe una producción científica afín que tributa a los propósitos generales de la UMET, respecto a elevar índices de Calidad de la Educación, tanto en su dimensión interna como externa, de modo que dichos autores, pudieran afiliarse a los grupos de trabajo, como un modo para continuar optimizando los resultados. Estas dimensiones comprenden, entre otras, el enfoque en temas de salud (prevención), prácticas pre-profesionales y habilidades investigativas.

Los proyectos de investigación enunciados al principio, han generado, en el lapso de que se analiza, una importante producción científica y como ente dinámico, ha ganado una creciente capacidad de convocatoria institucional, presente tanto, en la producción de publicaciones como en las contribuciones metodológicas, la cooperación interprofesional que aportan valoraciones múltiples, sobre los problemas que afectan hoy la Gestión de la Calidad de la Educación.

\section{Referencias bibliográficas}

Alvarez, I., Macías, A., \& Bernal, R. (2019). Talleres de orientación educativa para las familias de niños/as que asisten al Guagua Centro Carcelén Mágico, Quito. Revista Metropolitana de Ciencias Aplicadas, 2(1), 62-70. http://remca.umet.edu.ec/index.php/REMCA/article/view/82

Chou Rodríguez, R., Valdés Guada, A., y Sánchez Gálvez, S. (2017). Programa de formación de competencias digitales en docentes universitarios. Revista Universidad y Sociedad, 9(1), 81-86. http://rus.ucf.edu.cu

Jiménez, J. C., \& Moreira, Y. (2019). La práctica universitaria inclusiva de la Universidad Metropolitana de Ecuador: una alternativa desde el bienestar estudiantil. Revista Metropolitana de Ciencias Aplicadas, 2, 166-173. http://remca.umet.edu.ec/index.php/REMCA/article/view/106

López Calvajar, G., Nelson, C., y Baute, M. (2017). La tarea docente integradora. Caso optimización del plan de producción. Revista Universidad y Sociedad, 9, 120-128. http://scielo.sld.cu/pdf/rus/v9n1/rus17117.pdf

López Calvajar, G., Toledo Rodríguez, O. Meza Salvatierra, J. (2017). La formación de habilidades investigativas de los estsudiantes a través del estudio de caso. Universidad y Sociedad, http://scielo.sld.cu/pdf/rus/v9n4/rus13417.pdf 


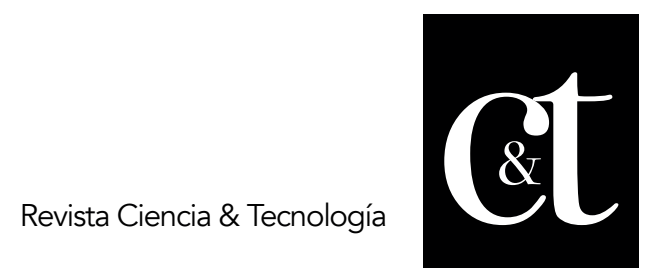

No. 27, 31 de julio de 2020

ISSN impreso: 1390 - 6321

ISSN online: 2661 - 6734

Millo , V., Fuentes, D., y González, V. E. (2017). Manual de procedimiento para el control interno en la universidad metropolitana. Revista Universidad y Sociedad, 9, 60-65. http://scielo.sld.cu/scielo.php?script=sci arttext\&pid=S2218$\underline{36202017000100008}$

Montero, C., Porraspita, D., y Hernández, D. (2016). La formación investigativa de los estudiantes, su organización y desarrollo en la Universidad Metropolitana del Ecuador (UMET). Revista Conrado, 12(53), pp. 14-18. https://conrado.ucf.edu.cu/index.php/conrado/article/view/301

Pérez Payrol, V. B., Baute Roales, M. y Luque Espinoza de los Monteros, M. (diciembre de 2017). La formación de la cultura ambiental en la carrera Ciencias de la Educación. Conrado, 13, 92-100. Recuperado el 10 de febrero de 2020, de https://conrado.ucf.edu.cu/index.php/conrado/article/view/653

Sánchez, V., López, M. M. y Baute, M. (2014). La práctica preprofesional en la carrera de Ciencias de la Educación de la Universidad Metropolitana del Ecuador. Universida y Sociedad, 7(1E), 74-79. http://rus.ucf.edu.cu/

Suárez, G., León, J. L., Morales, M. y Curbeira, D. (2019). Modelo para la formación de valores en la universidad inclusiva. Revista Conrado, 15(69), 79-88. http://scielo.sld.cu/pdf/rc/v15n69/1990-8644-rc-15-69-79.pdf

Tamayo, M., Gil, J.L., \& Carrillo, J. J. (2019). Pertinencia de la tutoría: carrera administración de empresas de la Universidad Metropolitana del Ecuador. Conrado, 15(68), 7-13. http://scielo.sld.cu/pdf/rc/v15n68/1990-8644-rc-1568-7.pdf

Toledo Rodríguez, O., Varela, M. y Meza Salvatierra, J. (2017). La Universidad Metropolitana de Ecuador: retos para los docentes de la carrera Contador Público Autorizado. Revista Conrado, 13 , https://conrado.ucf.edu.cu/index.php/conrado/article/view/645/6 